\title{
Effects of a fruit and vegetable intervention trial on net endogenous acid production: results from the US Women's Healthy Eating and Living (WHEL) Study
}

\author{
Manuela Abbate ${ }^{1}$, Yvonne Jeanes ${ }^{1}$, Susan Lanham-New ${ }^{2}$ and Lynda Frassetto ${ }^{3}$ \\ ${ }^{1}$ University of Roehampton, Roehampton, UK, ${ }^{2}$ University of Surrey, Surrey, UK and ${ }^{3}$ CTSI Clinical Research Centre, \\ San Francisco, USA
}

Homeostatic control of plasma $\mathrm{pH}$ is a tightly-regulated mechanism that can be strongly influenced by dietary habits. The increased ingestion of acid-forming foods characterising Western diets can induce a mild but chronic metabolic acidosis documented by increased renal net acid excretion (RNAE) ${ }^{(1)}$. Increased consumption of fruit and vegetables as part of an alkalizing diet has been observed to reduce net endogenous acid production (NEAP), calculated here as dietary protein: $\mathrm{K}$ intake and used as a valid indirect estimation of RNAE ${ }^{(1,2)}$.

This analysis has examined differences in nutrient intake and NEAP between the intervention arm of the US WHEL Study ( $n$ 79), who consumed eight to nine portions of fruit and vegetables daily (WHEL diet), and the matched control arm ( $n$ 93), who consumed three to five portions of fruit and vegetables daily (National Cancer Institute (NCI) diet) ${ }^{(3)}$ over the first 48 months of the study. According to the US Food and Drug Administration one portion of vegetables is equal to one cup of raw leafy vegetables, or half cup of other vegetables (cooked or chopped raw) or three-quarters cup of vegetable juice; one portion of fruit is equal to one medium apple, banana, orange or half cup chopped, cooked or canned fruit or three-quarters cup of fruit juice ${ }^{(4)}$.

FFQ data were collected at baseline and at 12 and 48 months for 179 women followed in the northern California site of the WHEL study. Data were analysed for intakes of dietary $\mathrm{Ca}, \mathrm{K}, \mathrm{Na}, \mathrm{P}$, protein and NEAP. Plasma $\alpha$ - and $\beta$-carotene concentrations were also measured, in concomitance with collection of FFQ, and used as markers of dietary compliance. Cross-sectional and longitudinal analyses were carried out using SPSS data analysis software (SPSS Inc., Chicago, IL, USA); all variables were tested for normality.

There were no differences in dietary composition, NEAP and plasma carotenoids concentrations between the groups at baseline. Both groups were compliant with dietary recommendations throughout the 48 -month period. Plasma $\alpha$ - and $\beta$-carotene concentrations were significantly higher in the WHEL group compared with the NCI group at both 12 months $(P<0.005)$ and 48 months $(P<0.005)$ after starting the dietary recommendations. The WHEL diet when compared with the NCI diet also provided higher intakes of Mg (mg/d) at both 12 months (372 (SD 94.1) and 329 (SD 88.2) respectively; $P=0.004$ ) and 48 months (352 (SD 97.8) and 301 (SD 85.9 ) respectively; $P=0.001)$ and $\mathrm{K}(\mathrm{mg} / \mathrm{d}$ ) at 48 months (3663 (SD 1055) and 2889 (SD 837) respectively; $P=0.001$ ). There were no differences in protein intakes between the two groups at any point in time. Increased levels of $\mathrm{K}$ and steady intakes of protein resulted in a greater decrease in NEAP (mEq/d) for the WHEL group compared with the NCI group at 12 months (-2166 (SD 524) and - 1695 (SD 459) respectively; $P=0.001)$ and 48 months ( -2004 (SD 596) and -1561 (SD 467) respectively; $P=0.001$ ).

Increasing dietary $\mathrm{K}$ intake by consuming eight to nine daily portions of fruit and vegetables decreases NEAP compared with eating three to five servings daily. Lowering dietary net acid loads has been shown to lower urine Ca excretion and may be important for bone and muscle health. Further analysis of the effect of estimated dietary acidity on indices of bone health is currently underway.

1. Frassetto LA, Todd KM, Curtis Morris R Jr \& Sebastian A (1998) Am J Clin Nutr 68, 576-583.

2. Lemann J, Bushinsky DA \& Hamm LL (2003) Am J Physiol Renal Physiol 285, 811-832.

3. National Cancer Institute (1995) 5 a Day for Better Health Program. Bethesda, MD: National Cancer Institute.

4. Food and Drug Agency (1997) Fruit and vegetables: eating your way to 5 a day. http://www.cfsan.fda.gov/ dms/fda5aday.html 